\title{
Research of Clustering Routing Algorithm in WSNs Based on Continuous Object
}

\author{
Die Hu, Guangcong Liu, Jifeng Chen \\ School of Computers, Guangdong University of Technology, Guangzhou 510006, China \\ hudie462588@163.com
}

Keywords: Wireless Sensor Networks, Bisecting K-means Algorithm, Continuous Object

\begin{abstract}
In monitoring application of continuous object that based on wireless sensor network, due to related parameters of continuous object in time and space are continuous, the data information that node acquired has strong correlation and continuity. On this basis,the idea that using bisecting K-means clustering algorithm to improve the traditional clustering routing algorithm is put forward. Simulation results show that in the monitoring application of continuous object,clustering algorithm that based on the bisecting K-means algorithm clustering algorithm is better than traditional clustering algorithm.It can make full use of node energy and balance the network energy better.
\end{abstract}

\section{Introduction}

Wireless sensor network ${ }^{[1]}$ is widely used in the fields of military, intelligent transportation and medical health and so on. In the application of target monitoring, the main research object is discrete object. But the monitoring of continuous object ${ }^{[2]}$ is an important aspect that can not be ignored in the application of target monitoring. The main features of the continuous object is the certain continuity in time and space of the related parameters. This article mainly discuss the continuous object.

Because of the relevant parameters in time and space of continuous object is continuous, so the node information that collected exist strong correlation and continuity. Leach clustering routing protocol is widely used in wireless sensor networks. But the clustering ${ }^{[3]}$ of Leach is according to the geographical location information of the node object, and the physical distribution and morphological feature of continuous object had not been taken into account .

Bisecting k-means algorithm ${ }^{[4]}$ has improved the k-means algorithm, effectively solves the problems caused by improper initial clustering center selection. Under the background of the monitoring application in continuous object, the use of bisecting k-means algorithm clustering can effectively balance the network energy ${ }^{[5]}$.

\section{Dynamic Modeling of Continuous Objects}

The main research of this paper is the case of instantaneous leakage, using the Gauss smoke model. In this paper, we will realize the evolution of continuous objects by shifting and superposition of the multiple Gauss smoke clusters. Without taking into account the diffusion of 3D, the diffusion model of Gauss smoke cluster is:

$$
c(x, y)=\frac{2 Q}{(2 \pi)^{3 / 2} \cdot \delta_{x} \delta_{y}} \cdot e^{-\frac{x^{2}}{2 \delta_{x}}} \cdot e^{-\frac{y^{2}}{2 \delta_{y}}}
$$

Among them: $\mathrm{C}(\mathrm{x}, \mathrm{y})$ is the concentration values of leak medium in somewhere at a certain time ; $\mathrm{Q}$ is the gas source intensity; $\operatorname{direct}(\mathrm{x})$ and $\operatorname{direct}(\mathrm{y})$ is the $\operatorname{diffusion}$ parameters in the direction of $\mathrm{x}$ and $\mathrm{y} ; \mathrm{x}$ and $\mathrm{y}$ is the coordinates of the value.

With time advancing, the smoke generated from different time periods can be translated and superimposed.To a certain extent, it can represent the evolution of continuous objects. Therefore, the distribution $\mathrm{D}(\mathrm{T})$ of the continuous object in $\mathrm{t}$ time: 


$$
\begin{gathered}
D(t)=\sum_{i=0}^{t} c(x, y)_{i} \\
c(x, y)_{i}=\frac{2 Q_{i}}{(2 \pi)^{3 / 2} \cdot \delta_{x i} \delta_{y i}} \cdot e^{-\frac{\left(x-u i \delta_{x i}\right)^{2}}{2 \delta_{x i}}} \cdot e^{-\frac{\left(x-u i \delta_{y j}\right)^{2}}{2 \delta_{y i}^{2}}}
\end{gathered}
$$

Where $\mathrm{t}$ is the units of time, $\mathrm{u}$ is the wind speed, and $\delta_{x i}$ 和 $\delta_{y i}$ is the diffusion parameters of $\mathrm{x}$ and y direction in i time. Qi is puff source of time $i$. The change process of gas diffusion is simulated in the following figure.


Fig. 1 The evolution of continuous object

Figure 1 is the diffusion situation of continuous object in the 5 seconds, 10 seconds and 30 seconds three time points , the diffusion direction is $(1,1),(0,1),(-1,1)$ and $(0,1)$. From Figure 1 can see that the continuous object spread with diffusion diffusion and concentration decrease with time.

\section{Bisecting K-means Clustering Algorithm}

Bisecting k-means clustering algorithm mainly improve the selection problem of the initial center for k-means clustering algorithm . Through literature [4], the main idea is: Firstly ,k-means clustering algorithm is used to divide the data set into 2 clusters, then add to the cluster M. Secondly, using k-means clustering algorithm to classify the cluster $\mathrm{Ci}$ that has the maximum SSE for I times under the premise of $\mathrm{k}$ equal to 2, called bisecting clustering. It will produce the minimum total SSE cluster and add it to the cluster $\mathrm{M}$. This cycle continues, until number of the cluster $\mathrm{M}$ reaches a given target number $\mathrm{K}$.

The initialize clustering center problem in bisecting k-means is relatively small. To cluster the cluster of maximum error SSE every time, so it is seen that the whole error function is convergence and choice of initial centers is weakly sensitive.

\section{Clustering}

\subsection{Clustering based on Bisecting K-means Algorithm}

Fully considering the relevance of data, the concentration distribution of gas diffusion is in the gradient distribution. In this paper, data are divided into cluster according to the continuous object distribution. In this simulation experiment, datas are divided into clusters in the area of $100 \mathrm{~m} *$ $100 \mathrm{~m}$, error parameters is 60 . Figure 2 is the concentration distribution of bisecting k-mean algorithm algorithm. Three color representation of three cluster, we can see the concentration gradient distribution of object object is corresponding from the graph.

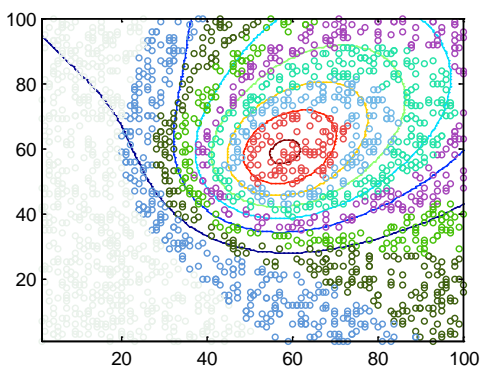

Fig.2 Bipartite k-means clustering algorithm results 


\subsection{Comparison of Bisecting k-means Clustering Algorithm and LEACH Clustering Algorithm}

From figure 2, we can see that bisecting k-means clustering algorithm give full consideration to the correlation of the sample data, and the final of the simulation results corresponding to the concentration distribution of continuous object.But Leach algorithm cluster nodes according to the geographic locations of the nodes only, and do not take into account the distribution characteristics of the object. Below will be compared to the two, as shown in figure 3 and figure 4 .

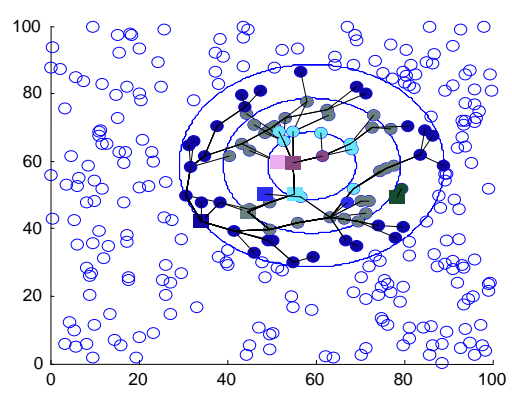

Fig.3 Network topology of bipartite k-means clustering algorithm

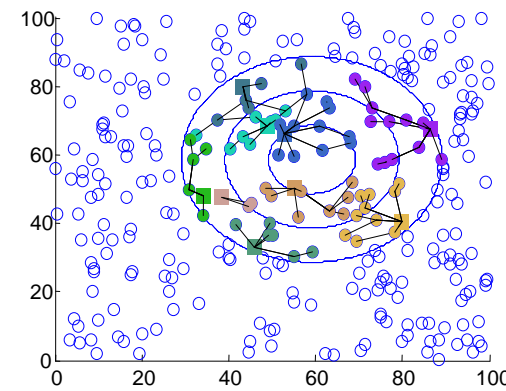

Fig.4 Network topology of LEACH

From the above comparison chart, clustering algorithm based Bisecting k-means clustering algorithm is superior to the traditional leach algorithm, because of its fully considering the distribution of the continuous object.

\section{Simulation and Analysis}

In order to validate the algorithm's validity. Simulation is carried out on the platform of Matlab.Simulation experiments using Gaussian puff model to simulate the continuous object.100 sensor nodes are randomly deployed to experiment scene. The simulation parameters are as follows: nodeCR is 10, Eelec is 50e-12, Emp is 1600e-9,simulateTime is 2000,baseStation is [0,0], alpha is 0.47 , beta is 0.53 . Figure 5 and figure 6 is comparative analysis of the algorithms in the network energy consumption and network node survival rate.

Figure 5 is the comparison of two algorithms' energy consumption in a period of time,it can be seen that the average energy of this algorithm in the 0 to 1600 time units is consumed in a linear form and steadily declined to 0.The traditional LEACH algorithm in the 0 to 1600 time units are steadily consumed to 0 , but the energy consumption of LEACH algorithm in the 0 to 1400 time is quick than this algorithm. Although in about 1400 time units, residual average energy of clustering algorithm of LEACH is higher than the this algorithm, but the number of nodes alive around $20 \%$ that can be seen in figure 6 . For Leach algorithm,at the end of the network cycle, there is still a part of the network node survival, but the energy of network did not get better use. The comparison shows that energy consumption in this algorithm is slow than the LEACH algorithm and the network energy has been better utilized. 


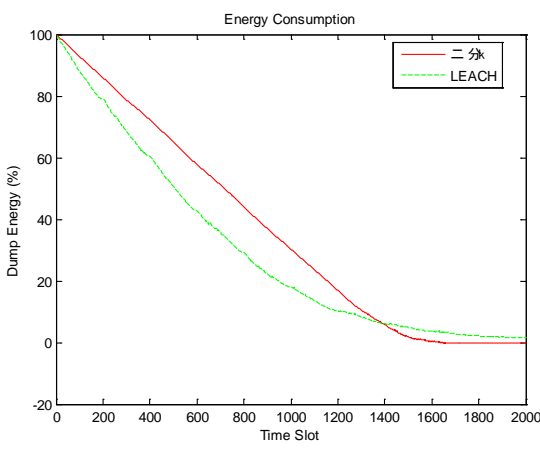

Fig.5 Dump energy

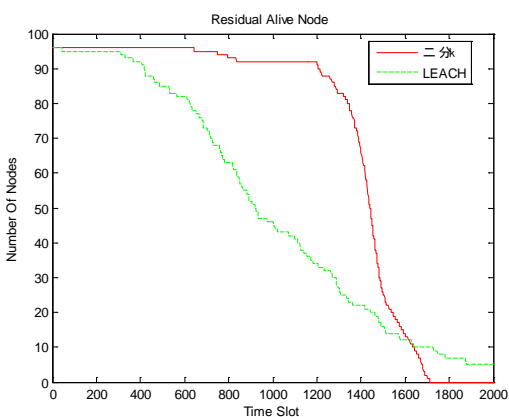

Fig.6 Data of survival nodes in network

Figure 6 is the analysis result of the survival number of nodes in the two algorithms. As can be seen from the chart, algorithm in this paper the number of death nodes increase rapidly after 1400 time units and die out at 1700 time units . In the traditional Leach algorithm, it have node death from 100 time units, until the 1650 time units, the number of live nodes is higher than the algorithm in this paper. But from figure 5, average energy is 0 at this time, and the the network cycle already end at the same time.So the remaining nodes can not be effective communication. A large number of holes appear in the network. The algorithm in this paper can guarantee a high survival rate of the nodes in a long period of time.It can balance the network energy effectively and improve the utilization rate of network energy.

\section{Summary}

In this paper, after study the clustering algorithm of continuous object, we propose new clustering idea that using bisecting k-means clustering algorithm clustering under the premise of fully take into account the correlation of the sample data in the continuous monitoring process. Difference in traditional leach algorithm,it consider nodes location information only. Through simulation and analysis, the clustering algorithm is proposed in this paper has greatly improved in balance the node energy consumption and network energy utilization rate.

\section{References}

[1] Cui L, Ju H L,Miao Y,et al. Advances in wireless sensor networks[J]. Computer Research and Development,2005,42(1):163-174.

[2] Tu S C, Chang G Y, Sheu J P, et al. Scalable continuous object detection and tracking in sensor networks[J]. Journal of Parallel and Distributed Computing, 2010, 70(3): 212-224.

[3] Cao J L, Chen Y C,Ren Z,et al.Of multiple rounds of the cluster-based routing protocol for wireless sensor networks[J].Computer Science, 2013,40(7):67-70.

[4] Liu G C,Huang T T,Chen H N. Improved bisecting K-means Clustering Algorithm[J].Computer Application and Software,2015,32(2):261-264.

[5] Lü T, Zhu Q X, Zhu Y Y. An energy balance algorithm for clustering in wireless sensor networks [ J ]. Journal of Computer Application, 2012,32(11):3107-3111. 